\title{
Investigation of PDMS as coating on CMUTs for Imaging
}

la Cour, Mette Funding; Stuart, Matthias Bo; Laursen, Mads Bjerregaard; Diederichsen, Søren Elmin; Thomsen, Erik Vilain; Jensen, Jørgen Arendt

\section{Published in:}

Proceedings of IEEE International Ultrasonics Symposium 2014

Link to article, DOI:

10.1109/ULTSYM.2014.0645

Publication date:

2014

Document Version

Early version, also known as pre-print

Link back to DTU Orbit

Citation (APA):

la Cour, M. F., Stuart, M. B., Laursen, M. B., Diederichsen, S. E., Thomsen, E. V., \& Jensen, J. A. (2014). Investigation of PDMS as coating on CMUTs for Imaging. In Proceedings of IEEE International Ultrasonics Symposium 2014 (pp. 2584-2587). IEEE. https://doi.org/10.1109/ULTSYM.2014.0645

\section{General rights}

Copyright and moral rights for the publications made accessible in the public portal are retained by the authors and/or other copyright owners and it is a condition of accessing publications that users recognise and abide by the legal requirements associated with these rights.

- Users may download and print one copy of any publication from the public portal for the purpose of private study or research.

- You may not further distribute the material or use it for any profit-making activity or commercial gain

- You may freely distribute the URL identifying the publication in the public portal 
Paper presented at the IEEE International Ultrasonics Symposium:

\section{Investigation of PDMS as coating on CMUTs for imaging}

Mette Funding la Cour, Matthias Bo Stuart, Mads Bjerregaard Laursen, Søren Elmin Diederichsen, Erik Vilain Thomsen and Jørgen Arendt Jensen

Center for Fast Ultrasound Imaging, Biomedical Engineering Group, Department of Electrical Engineering Ørsteds Plads Building 349,

Technical University of Denmark, 2800 Kgs. Lyngby, Denmark. 


\title{
Investigation of PDMS as coating on CMUTs for imaging
}

\author{
Mette Funding la Cour*†, Matthias Bo Stuart*, Mads Bjerregaard Laursen ${ }^{\dagger}$, \\ Søren Elmin Diederichsen ${ }^{\dagger}$, Erik Vilain Thomsen ${ }^{\dagger}$ and Jørgen Arendt Jensen* \\ ${ }^{*}$ Center for Fast Ultrasound Imaging, Department of Electrical Engineering, Technical University of Denmark, \\ DK-2800 Kgs. Lyngby, Denmark \\ ${ }^{\dagger}$ Department of Micro and Nanotechnology, Technical University of Denmark, DK-2800 Kgs. Lyngby, Denmark
}

\begin{abstract}
A protective layer is necessary for Capacitive Micromachined Ultrasonic Transducers (CMUTs) to be used for imaging purpose. The layer should both protect the device itself and the patient while maintaining the performance of the device. In this work Sylgard 170 PDMS is tested as coating material for CMUTs through comparison of transmit pressure and receive sensitivity in immersion of coated and uncoated elements. It is seen that the transmitted pressure decreases with $27 \%$ and the receive sensitivity decreases $35 \%$ when applying the coating using a dam and fill principle. This matches well with the estimated value of $31 \%$. With the coating, the center frequency was found to be decreased from 4.5 MHz to 4.1 $\mathrm{MHz}$ and the fractional bandwidth was increased from $77 \%$ to $84 \%$ in transmit. In receive the center frequency was found to decrease from $4.4 \mathrm{MHz}$ to $3.9 \mathrm{MHz}$ and the fractional bandwidth was decreased from $108 \%$ to $92 \%$, when applying the PDMS coating.
\end{abstract}

\section{INTRODUCTION}

Coating of Capacitive Micromachined Ultrasonic Transducers (CMUTs) is important for insulation between the surface of the elements and the patient, when applying the high voltages required for operating CMUTs. Furthermore it also protects the surface of the device against environmental factors and e.g. degradation of the electrodes [1].

A possible coating material should have good acoustical properties such that the impedance matches with the medium for high energy transfer and a glass transition temperature below room temperature providing a low static Young's modulus for preserving the CMUT's pull-in voltage [2]. Furthermore, the coating needs to be biocompatible. Polydimethylsiloxane (PDMS), Sylgard 170, is chosen as coating material in this work since it fulfills these requirements. Its acoustical properties match well with water and tissue (acoustical impedance 1.5 MRayls for water, 1.63 MRayls for tissue and 1.37 MRayls for Sylgard 170).

Other coating materials have previously been investigated e.g. Parylene C [1], [3], which gives good results and has the advantage of being cleanroom compatible, but is deposited using Vapor Deposition Polymerization (VDP). Silicon nitride has also been proposed due to cleanroom compatibility, however, the stress in the nitride highly affects the device performance [4]. Different types of PDMS have also been investigated, and it is seen that some will increase the output signal, due to increased mass loading, and others will decrease the influence of the echo from the coating-water interface, due to better impedance matching [2], [3]. Many of the experiments regarding coating have been conducted in air using a vibrometer, and thus need

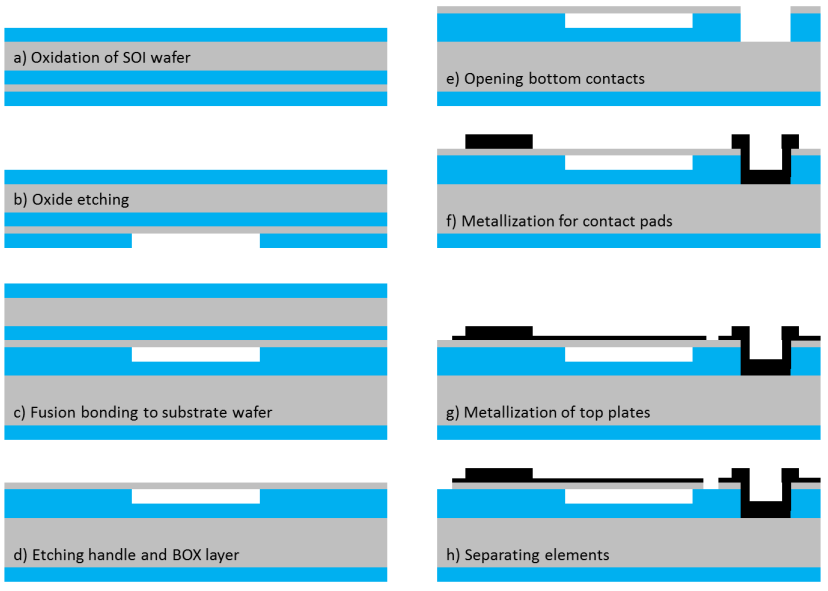

Fig. 1. Process steps for fabricating 1D arrays using fusion bonding and oxidation of both SOI and substrate wafer. Both top and bottom electrode can be contacted from the front side of the device.

further testing to check the influence on performance for imaging.

The objective for this work is to investigate how the Sylgard 170 PDMS coating affects the CMUT performance through comparison of the transmit pressure and receive sensitivity for devices with and without coating.

\section{TRANSDUCER FABRICATION}

To test whether the Sylgard 170 PDMS is a suitable coating material for CMUTs, 128 element 1D arrays were fabricated with a fusion bonding process. The overall process flow can be seen in Fig. 1. This process is developed to minimize the number of process steps, while avoiding bumps at the corners [5]. Bumps on the oxide surface often arise from having two oxidations of the substrate wafer to form cavities and an insulation layer separately. However, the bumps can ruin the fusion bonding quality and the double oxidation method then requires an extra etching step to etch back the bumps. The first step is to oxidize the silicon-on-insulator (SOI) wafer and etch cavities in the oxide. An oxidation is performed on the substrate wafer as well to obtain an insulation layer in the bottom of the cavities. Fusion bonding is performed and followed by high temperature annealing. The handle layer and buried oxide layer are etched away before opening up to the bottom electrode. A thick aluminum layer $(800 \mu \mathrm{m})$ is deposited for bonding pads 


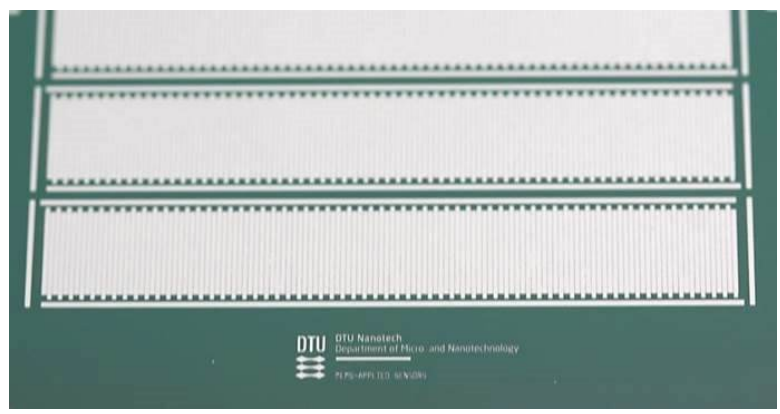

Fig. 2. Photo of fabricated 1D CMUT arrays after end of fabrication. The arrays have 128 elements and are designed to operate at $5 \mathrm{MHz}$.

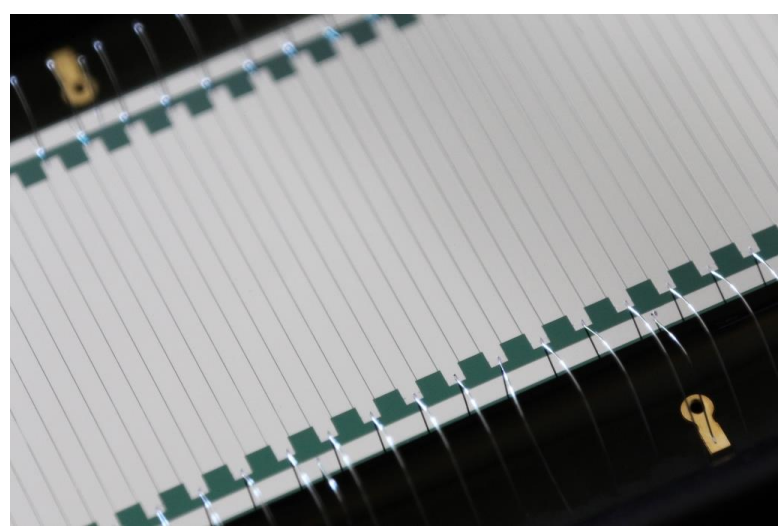

Fig. 3. Photo of finished 1D array mounted on and wirebonded to a PCB. The elements are seen as the vertical metal lines where every second has a contact pad to the same side of the array. The bottom contact is seen as a metal bar along the array.

and a thin aluminum layer $(200 \mu \mathrm{m})$ is deposited to completely cover the top electrodes. The top plates and elements are defined by etching aluminum and silicon. A picture of a finished array can be seen in Fig. 2. The elements have contact pads at the ends and the bottom contact for reaching the substrate is running along the length of the array. Fig. 3 shows a microscope picture of an array, where the elements can be seen as vertical lines, and every second element has contact pads to the same side. The thicker aluminum layer at the pads improves the wirebonding.

The arrays are aimed at an immersion resonant frequency of $5 \mathrm{MHz}$. They are linear arrays with a $\lambda$ pitch i.e. $300 \mu \mathrm{m}$. Each element consists of 460 square shaped cells with a side length of $49 \mu \mathrm{m}$ and is $5 \mathrm{~mm}$ long.

\section{DEVICE COATING AND MEASUREMENT SETUP}

There are several ways to apply a coating to a transducer: mold-transfer [2], spray coating, VDP [1], [3], and spin coating [3]. For CMUTs insulating layers are usually applied using mold-transfer to integrate a lens at the same time. However, a lens should not be applied for this application as the uncoated devices used for comparison will not be focused. Spray and spin coating are better for wafer scale coating, so instead an alternative method was used. This method will now be described in detail.

To test the PDMS coating, one of the fabricated arrays was

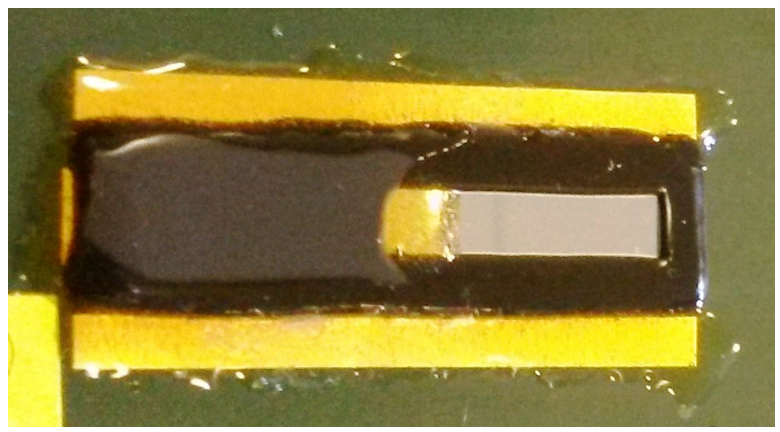

Fig. 4. CMUT array mounted on a PCB with PDMS coating applied to half of the array using the glob top and an epoxy as a dam.

mounted on a printed circuit board (PCB) and wirebonded. The wirebonds are covered by a protective glob top (CHIPCOAT G8345D) and this is used as a dam when applying the PDMS coating. The dam is filled with liquid PDMS and then cured in vacuum. The procedure for this PDMS coating is

- Mix the two components of Sylgard 170 and de-gas in a vacuum chamber for $20 \mathrm{~min}$

- Apply to device using a syringe with a needle tip by dripping the PDMS onto the surface at a close distance

- De-gas the coated array in a vacuum chamber for 60 $\min$

- Cure in a $70^{\circ} \mathrm{C}$ oven for at least 1 hour

The height of the glob top dam and thus also the thickness of the coating is estimated to be $\sim 900 \mu \mathrm{m}$. Coating thickness have been investigated by Lin et al. [2], who found that the main signal is not affected by the thickness. However, if the coating is thin, the echo from the coating-liquid interface will influence the spectrum. According to their results, this should not be a problem with this thickness of coating.

For this particular experiment of coating evaluation, it was desired to have the coated and uncoated elements as similar to each other as possible. Therefore, half of an array was coated using the described method and the other half was left without coating. A picture of the half coated device can be seen in Fig. 4.

For evaluating the transducers with and without coating, a flexible platform developed for testing different CMUTs was used. The layout of the setup can be seen in Fig. 5, where the transducer is mounted on and wirebonded to PCB1. This PCB is clicked onto another PCB containing all the electronics for operating the CMUT, and this second PCB can be reused for other devices. A transducers cable for a BK Medical scanner is also attached to PCB2, so the transducer can be connected to an imaging system. A picture of the setup can be seen in Fig. 6.

\section{MEASUREMENTS AND DISCUSSION}

The measurements are performed with the experimental Synthetic Aperture Real-time Ultrasound System (SARUS) [6]. All measurements are performed in oil for electrical insulation of the uncoated part of the device. Acoustical 


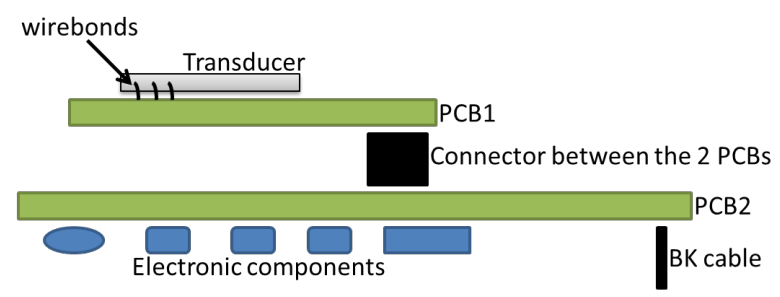

Fig. 5. Sketch of principle of transducer evaluation platform for testing various CMUT designs and chips. The CMUT is wirebonded to a PCB, which is connected to a second PCB with a transducer cable attached.

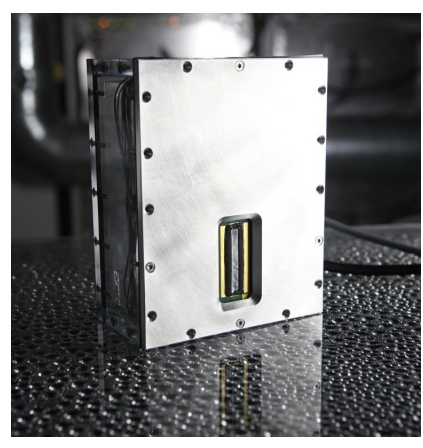

(a) Frontside.

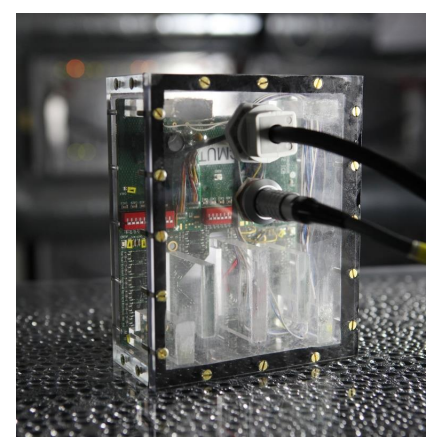

(b) Backside.
Fig. 6. Flexible transducer evaluation platform developed to test multiple CMUT designs and devices. The front side is open to the CMUT and on the backside the transducer cable and a separate DC supply cable can be seen.

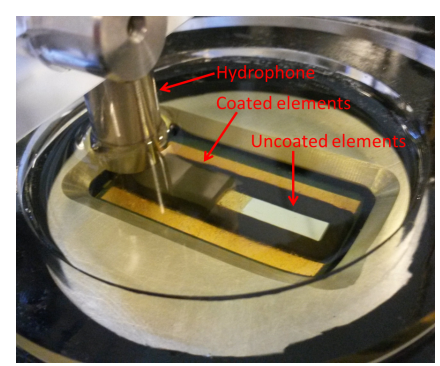

(a) Hydrophone setup.

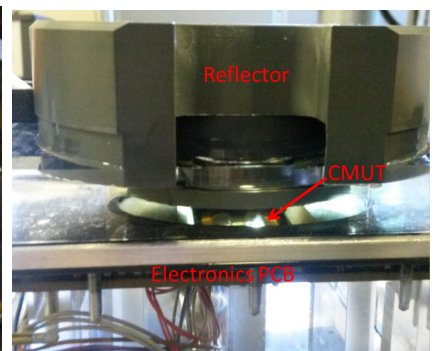

(b) Plane reflector setup.
Fig. 7. Measurement setup using hydrophone and plane reflector in front of the CMUT mounted in the box with electronics.

measurements are made to obtain the transmitted pressure and the receive sensitivity for the two halves of the device. Ten working elements are chosen on each half of the array for the experiments. For all measurements the transducer elements are biased at $190 \mathrm{~V}$, which is $80 \%$ of the calculated pull-in voltage. The AC transmit signal is $\pm 60 \mathrm{~V}$.

\section{A. Transmit pressure}

A hydrophone (Optel $5 \mathrm{MHz}$, Optel, Wroclaw, Poland) placed $10 \mathrm{~mm}$ from the transducer surface is used to measure the transmit pressure, as seen in Fig. 7a. Ten different white, Gaussian random signals are used for the excitation. The RMS of the sampled signals is calculated and averaging is done over the 10 random signals. The hydrophone is aligned to the center of all elements when measuring across the array. The values

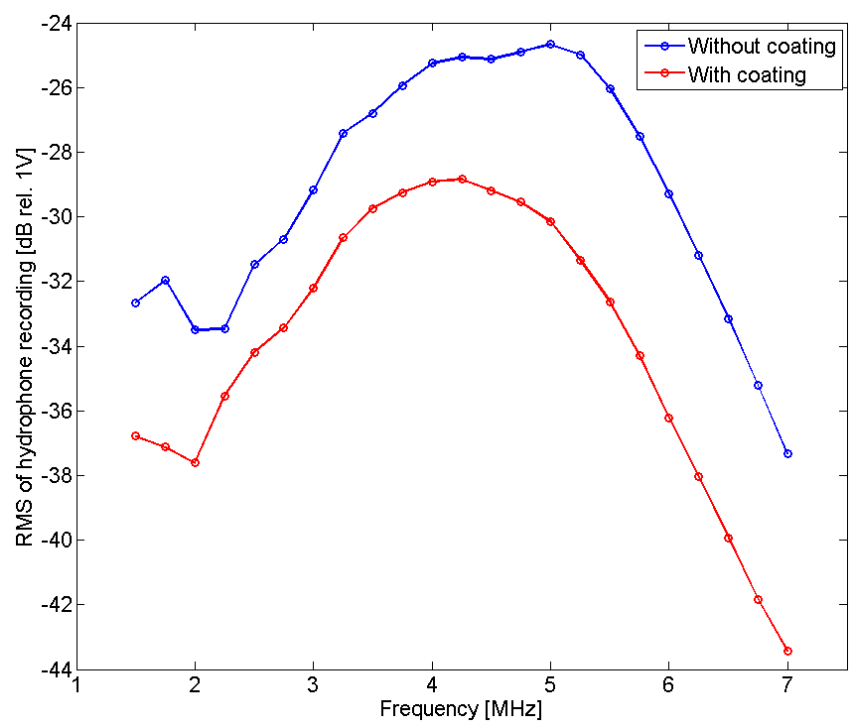

Fig. 8. Transfer function in transmit for elements with and without coating found as a mean of 10 elements of each kind. An 8 pulse, narrowband excitation is used for each frequency.

are an average of 10 working elements with or without coating applied. For the transmitted pressure it was found that the array with coating has an output signal of $27 \%$ less than the array without coating.

The attenuation in PDMS can be described as [2]

$$
L_{\mathrm{dB}}=\alpha f^{\beta} w \text { or } \frac{V(w)}{V_{0}}=10{ }^{\left(-\frac{\alpha f^{\beta}}{20}\right)},
$$

where $I_{0}$ is the original intensity, $\alpha$ the attenuation loss factor (given in $\mathrm{dB} / \mathrm{MHz} / \mathrm{mm}$ ), $f$ the frequency, $\beta$ an empirically found parameter and $w$ the thickness of the coating. For the Sylgard $170 \mathrm{PDMS}, \alpha=0.37 \mathrm{~dB} / \mathrm{MHz} / \mathrm{mm}$ and $\beta=1.4$. Using a frequency of $5 \mathrm{MHz}$ and the estimated coating thickness of $0.9 \mathrm{~mm}$, the expected drop in signal amplitude is $31 \%$, which is comparable to the measured signal loss with a difference of $\pm 13 \%$ respectively for transmit and receive. The differences could arise from the estimation of coating thickness.

Hydrophone measurements were also performed for varying frequencies. A narrowband, 8 period, excitation was used at each frequency. The frequency sweep was made from $1.5 \mathrm{MHz}$ to $7 \mathrm{MHz}$ in steps of $250 \mathrm{kHz}$. The hydrophone was placed at the center of each element at a distance of $10 \mathrm{~mm}$ and the average results for 10 elements with and without coating can be seen in Fig. 8. From this the mean center frequency is found to be $4.5 \mathrm{MHz}$ for the elements without coating and 4.1 $\mathrm{MHz}$ for the elements with coating. The coating results in a decrease in center frequency of around $9 \%$, which is due to the added mass on the plate. Similarly the fractional bandwidth is found to be $77 \%$ for the array without coating and $84 \%$ with the coating. Thus, the PDMS coating slightly increases, $9 \%$, the fractional bandwidth when transmitting pressure, which is explained by the increased dampening of the plate. It is also seen that applying this coating with a thickness of $0.9 \mathrm{~mm}$ results in a loss in signal of around $3.8 \mathrm{~dB}$ at the center frequency. 


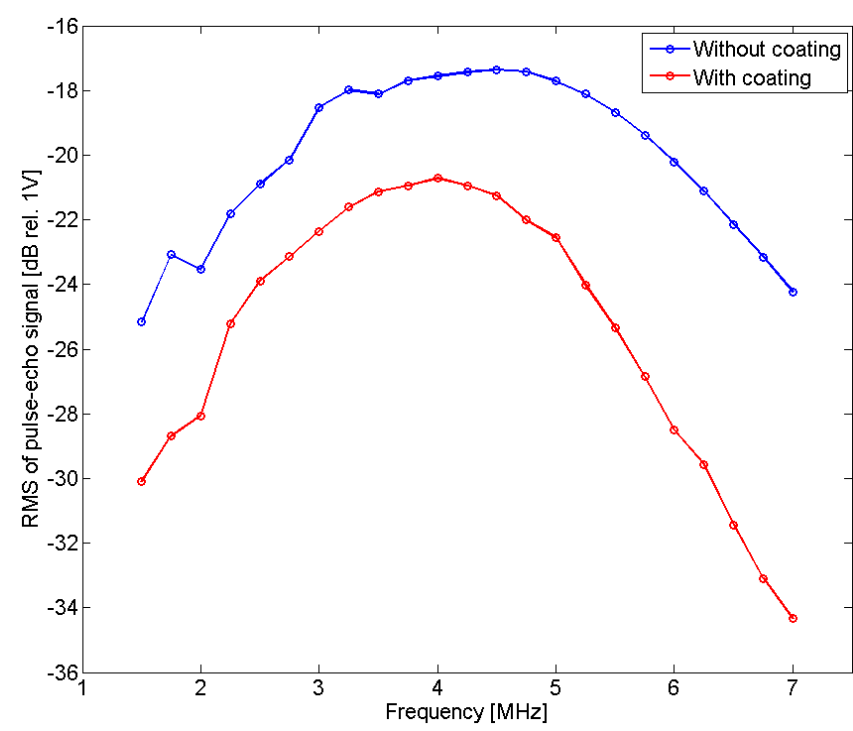

Fig. 9. Transfer function in receive for elements with and without coating found as a mean of 10 elements of each kind. An 8 pulse, narrowband excitation is used for each frequency.

\section{B. Receive sensitivity}

To measure receive sensitivity a plane reflector of $40 \mathrm{~mm}$ PVC (Polyvinylchloride) was placed at a distance of $10 \mathrm{~mm}$ from the transducer surface as seen in Fig. 7b. Again ten different white, Gaussian random signals are used for the excitation and the RMS of the sampled signal is calculated. Averaging is done over the 10 random signals.

The results from the receive analysis showed a decrease of $35 \%$ for the coated elements compared to the uncoated which matches well with the expected value found from (1). The receive sensitivity is found by dividing the measured pulseecho signal with the measured transmit pressure for the same element to take into account that the coated elements also transmit less pressure than the elements without coating.

A pulse-echo analysis to find the transfer function using a plane reflector was also carried out. The same method as for the transmit analysis was used and a narrowband, 8 period excitation applied. Again, a frequency sweep was made from $1.5 \mathrm{MHz}$ to $7 \mathrm{MHz}$ in steps of $250 \mathrm{kHz}$, and the average result for 10 elements of each kind can be seen in Fig. 9. This shows the pulse-echo frequency characteristic. It is seen that the loss in signal is around $3.4 \mathrm{~dB}$. The $-6 \mathrm{~dB}$ center frequency and fractional bandwidth were found again from the normalized pulse-echo signal. This resulted in the center frequency being 4.4 $\mathrm{MHz}$ and $3.9 \mathrm{MHz}$ for the elements without and with coating, respectively. This means a decrease of $11 \%$. The measured fractional bandwidths were found to be $108 \%$ and $92 \%$, respectively, resulting in a decrease of $15 \%$. Again, the coating decreases the center frequency due to the added mass and the fractional bandwidth is decreased as well, which can also be ascribed to the added mass.

The results from the frequency sweep measurements for both transmit and receive are summarized in Table I.
Table I. RESULTS FOR CENTER FREQUENCY AND FRACTIONAL BANDWIDTH FROM HYDROPHONE AND PLANE REFLECTOR MEASUREMENTS OF 10 ELEMENTS WITH AND 10 ELEMENTS WITHOUT COATING.

\begin{tabular}{cccc}
\hline \hline Measurement & Without coating & With coating & Difference \\
\hline Center frequency, transmit & $4.5 \mathrm{MHz}$ & $4.1 \mathrm{MHz}$ & $-9 \%$ \\
Fractional bandwidth, transmit & $77 \%$ & $84 \%$ & $+9 \%$ \\
Center frequency, receive & $4.4 \mathrm{MHz}$ & $3.9 \mathrm{MHz}$ & $-11 \%$ \\
Fractional bandwidth, receive & $108 \%$ & $92 \%$ & $-15 \%$ \\
\hline \hline
\end{tabular}

\section{Conclusion}

The initial measurements performed on the two devices show that the $0.9 \mathrm{~mm}$ thick Sylgard 170 PDMS coating decrease the performance of the CMUT array around $30 \%$ regarding transmitted pressure and receive sensitivity. In both transmit and pulse-echo measurements the transfer function was found by sweeping the frequency and a decrease of the center frequency of $9-11 \%$ was found. The fractional bandwidth was found to increase by $9 \%$ in transmit and decrease by $15 \%$ in receive. The losses in $\mathrm{dB}$ was found to be around $3.8 \mathrm{~dB}$ in transmit and $3.4 \mathrm{~dB}$ in pulse-echo. In conclusion, some effects are always expected from a coating due to the loss in the material and with the measured influence of the Sylgard 170 PDMS, this is a good option for coating of CMUTs.

\section{ACKNOWLEDGEMENT}

This work was financially supported by the Danish National Advanced Technology Foundation (024-2008-3) and (82-20124).

\section{REFERENCES}

[1] E. Jeanne, C. Meynier, J. Terry, M. Roy, L. Haworth, and D. Alquier, "Evaluation of parylene as protection layer for capacitive micromachined ultrasonic transducers," ECS Transactions, vol. 11, no. 16, pp. 25-33, Mar. 2008.

[2] D.-S. Lin, X. Zhuang, S. H. Wong, M. Kupnik, and B. T. Khuri-Yakub, "Encapsulation of capacitive micromachined ultrasonic transducers using viscoelastic polymer," Journal of microelectromechanical systems : a joint IEEE and ASME publication on microstructures, microactuators, microsensors, and microsystems, vol. 19, no. 6, pp. 1341-1351, Dec. 2010.

[3] X. Zhuang, A. Nikoozadeh, M. A. Beasley, G. G. Yaralioglu, B. T. KhuriYakub, and B. L. Pruitt, "Biocompatible coatings for CMUTs in a harsh, aqueous environment," Journal of Micromechanics and Microengineering, vol. 17, no. 5, p. 994, May 2007.

[4] E. Jeanne, C. Meynier, F. Teston, D. Certon, N. Felix, M. Roy, and D. Alquier, "Protection layer influence on capacitive micromachined ultrasonic transducers performance," MRS Online Proceedings Library, vol. 1052, 2007.

[5] T. L. Christiansen, O. Hansen, M. D. Johnsen, J. N. Lohse, J. A. Jensen, and E. V. Thomsen, "Void-free direct bonding of CMUT arrays with single crystalline plates and pull-in insulation," in Ultrasonics Symposium (IUS), 2013 IEEE International, Jul. 2013, pp. 1737-1740.

[6] J. A. Jensen, H. Holten-Lund, R. T. Nilsson, M. Hansen, U. D. Larsen, R. P. Domsten, B. G. Tomov, M. B. Stuart, S. I. Nikolov, M. J. Pihl, Y. Du, J. H. Rasmussen, and M. F. Rasmussen, "SARUS: A synthetic aperture real-time ultrasound system," IEEE Transactions on Ultrasonics, Ferroelectrics, and Frequency Control, vol. 60, no. 9, pp. 1838-1852, Sep. 2013. 